\title{
A New Method for Estimating Inverse Data from Destructive Regular Storage Life Test
}

\author{
H. Wang, X.B. Ma \\ School of Reliability and Systems Engineering \\ Beihang University \\ Beijing, China
}

\begin{abstract}
In the destructive regular storage life test of products, there is "inverse" data sometimes, resulting in inaccurate estimates of its reliability index. Based on the theory of isotonic regression and minimum chi-square estimation, we proposed a new method for processing "inverse" data. First, alter the possible "inverse" of original frequency into the frequency meeting sequence constraint by using PAVA algorithm. Then, estimate reliability parameters by means of minimum chi-square estimation. Comparing with traditional methods, we increased the distribution-test of overall failure probability function, the point estimates and confidence intervals of reliability parameters during storage period of products. Finally, example shows that the coefficient of variation obtained by this method are smaller than MLE and the coefficient of variation changes little when sample size changes, reflecting its superiority for estimating small sample data.
\end{abstract}

Keywords- inverse data; isotonic regression; minimum chisquare estimation; coefficient of variation

\section{INTRODUCTION}

Under normal circu mstances, the products after produced in the factory, for some reasons need to be stored in the warehouse for some time. Due to the effect of storage conditions, products' reliability indices will decline. To identify the change of the quality of products at different stages of the storage period, we need to test products during storage period and analyze test data. Usually destructive regular storage life test is carried out as follows:

Mark $t=\left(t_{1}, t_{2}, \cdots, t_{k}\right)$ as the test time, after a storage time $t_{i}$, extract $n_{i}$ products and we can find $X_{i}$ products which are failed. Then we obtain the data:

$$
\left(t_{i}, n_{i}, X_{i}\right), \quad i=1,2, \cdots, k
$$

in which, $0<t_{1}<t_{2}<\cdots<t_{k}$. All tests are independent.

In practice, by the time and cost constraints, etc. The sample size $n_{i}$ is usually small, so the test results will be seriously affected by the randomness of the samples. And sometimes it will come as $X_{i+1} / n_{i+1} \square X_{i} / n_{i}$, which does not meet the characteristic that the probability of failure reduces with time increasing. Such data is always called "inverse" data [8] in engineering, and the estimate result calculated by this would seriously have a great bias.

To solve the problem above and regulate "inverse" data, scholars do a lot of related work, like the "inverse" data estimating method proposed in [9, 10] based on Bayes theory. But Bayes method requires the prior distribution information of products' life, which has limited applications. In this paper the idea of is otonic regression $[1,3,6]$ is applied to analyzing data from the storage life test, regulating the inversed frequency of failure due to the randomness. And then, combined with Pearson goodness of fit theory, we give a complete method for estimating test data.

\section{ISOT ONIC REGRESSION BASED ON FAILURE FREQUENCY}

In the destructive regular storage life test, the failure frequency at time $t_{i}$ is:

$$
f_{i}=\frac{X_{i}}{n_{i}}, \quad i=1,2, \cdots, k
$$

We assume $T=\left\{t_{1}, t_{2}, \cdots, t_{\mathrm{k}}\right\}$ is a finite set, and $f=\left(f_{1}, f_{2}, \cdots, f_{k}\right)^{\prime}$ is a bounded function defined on $T$. Also we define a semi order " $\prec$ ” on $T$, when:

$$
t_{i} \in T, t_{j} \in T, t_{i} \prec t_{j}
$$

the inequality $f_{i}^{*}=f^{*}\left(t_{i}\right) \leq f_{j}^{*}=f^{*}\left(t_{j}\right)$ is always workable, then we call $f^{*}=\left(f_{1}^{*}, f_{2}^{*}, \cdots, f_{k}^{*}\right)^{\prime}$ is an order-preserving function relative to" $\prec$ "defined on $\mathrm{T}$.

Mark $\mathrm{G}$ as the whole set for the order-preserving functions. If there is $f^{*} \in G$, which satisfied with:

$$
\sum_{i=1}^{k}\left(f_{i}-f_{i}^{*}\right)^{2} \omega_{i}=\min _{\forall g \in G} \sum_{i=1}^{k}\left(f_{i}-g_{i}\right)^{2} \omega_{i}
$$

then we call $f^{*}=\left(f_{1}^{*}, f_{2}^{*}, \cdots, f_{k}^{*}\right)^{\prime}$ as an isotonic regression of $f$, when $\omega=\left(\omega_{1}, \omega_{2}, \cdots, \omega_{k}\right)^{\prime}, \omega_{i}>0$ is a weight function. By using the calculation method of isotonic regression below, we can alter the possible "inverse" of original frequency into the frequency meeting sequence constraint.

Algorithm PA VA:

a. If $f \in G$, then $f^{*}=f$; 
b. If there is a $j$ making $f_{j}>f_{j+1}$, we make:

$$
B=\{j, j+1\}, \quad f_{B}=A_{V}(B)=\frac{\sum_{i \in B} f_{i} \omega_{i}}{\sum_{i \in B} \omega_{i}}, \quad \omega_{B}=\omega_{j}+\omega_{j+1}
$$

Moreover:

$$
\begin{aligned}
& \tilde{f}=\left(f_{1}, \cdots, f_{j-1}, f_{B}, f_{j+2}, \cdots, f_{k}\right)^{\prime} \\
& \tilde{\omega}=\left(\omega_{1}, \cdots, \omega_{j-1}, \omega_{B}, \omega_{j+2}, \cdots, \omega_{k}\right)^{\prime}
\end{aligned}
$$

c. Repeat step b until subscript set $\mathrm{K}$ is divided into $l$ pieces: $B_{1}, B_{2}, \cdots, B_{l}$, which is satisfied with:

$$
A_{V}\left(B_{1}\right)<A_{V}\left(B_{2}\right)<\cdots<A_{V}\left(B_{l}\right)
$$

Then we can get:

$$
f_{i}^{*}=A_{V}\left(B_{t}\right), \quad i \in B_{t}, \quad i=1,2, \cdots, l
$$

III. PEARSON CHI-SQUARE GOODNESS OF FIT TEST WITH VARIABLE SAMPLES AND MINIMUM CHI-SQUARED ESTIMATION

In the theory of goodness of fit test [2,4], Pearson proposed $\chi^{2}$ statistics used to test and verify the joint distribution of a group of independent samples whether belonging to the family of distributions with specific properties. Among these, the form of composite variable sample Pearson $\chi^{2}$ statistic is:

$$
\chi^{2}(\vec{\lambda})=\sum_{i=1}^{k} \frac{\left(X_{i}-n_{i} p_{i}(\vec{\lambda})\right)^{2}}{n_{i} p_{i}(\vec{\lambda})}
$$

in which, $\vec{\lambda}=\left(\lambda_{1}, \lambda_{2}, \cdots\right)$ represents parameter vector.

Pearson $\chi^{2}$ statistic describes the difference between desired frequencies and observed frequencies. When $n_{i} \rightarrow \infty$, the limit distribution of $\chi^{2}(\vec{\lambda})$ is $\chi^{2}$ distribution whose degree of freedom is $k-1$, that is $\chi^{2}(\vec{\lambda}) \sim \chi_{k-1}^{2}$. When conducting the test, without knowing the true value of $\vec{\lambda}$, a natural thought is to replace $\vec{\lambda}$ with the estimated value of $\vec{\lambda}$, which is $\hat{\vec{\lambda}}$. To calculate $\chi^{2}(\vec{\lambda})$, we use:

$$
\chi^{2}(\hat{\vec{\lambda}})=\sum_{i=1}^{k} \frac{\left(X_{i}-n_{i} p_{i}(\hat{\vec{\lambda}})\right)^{2}}{n_{i} p_{i}(\hat{\vec{\lambda}})}
$$

as the test statistic, Fisher proves that when $\hat{\vec{\lambda}}$ is the consistency estimation of $\vec{\lambda}$, the limit distribution of $\chi^{2}(\vec{\lambda})$ is $\chi_{k-s-1}^{2}$, when $s$ is the dimension of parameter $\vec{\lambda}$. So if significance level is $\alpha$, we have:

$$
P\left(\chi^{2}(\hat{\vec{\lambda}}) \geq \chi_{k-s-1}^{2}(1-\alpha) \mid H_{0}\right) \leq \alpha
$$

When $\chi^{2}(\hat{\vec{\lambda}}) \geq \chi_{k-s-1}^{2}(1-\alpha)$, small probability events will reject the null hypothesis.
The meaning of minimu m chi-square estimation is that we consider the value $\tilde{\lambda}$ which minimizes Pearson $\chi^{2}$ statistic as the best estimated value of $\vec{\lambda}$, which is:

$$
\chi^{2}(\tilde{\lambda})=\min \left\{\chi^{2}(\vec{\lambda}): \vec{\lambda} \in \Lambda\right\}
$$

Fisher also points out that $\tilde{\lambda}$ and $\hat{\vec{\lambda}}$ are equivalent, in other words, $\chi^{2}(\tilde{\lambda})$ and $\chi^{2}(\hat{\vec{\lambda}})$ share the same limit distribution.

Solve the equation $\frac{\partial \chi^{2}(\vec{\lambda})}{\partial \lambda_{j}}=0, \quad j=1,2, \cdots, s$, we can get the minimu m chi-square estimation of $\vec{\lambda}$.

$$
\begin{aligned}
\frac{\partial \mathcal{X}^{2}(\vec{\lambda})}{\partial \lambda_{j}} & =\sum_{i=1}^{k}\left\{-\frac{2 n_{i}\left(X_{i}-n_{i} p_{i}(\vec{\lambda})\right)}{n_{i} p_{i}(\vec{\lambda})}-\frac{\left(X_{i}-n_{i} p_{i}(\vec{\lambda})^{2}\right.}{n_{i} p_{i}^{2}(\vec{\lambda})}\right\} \cdot \frac{\partial p_{i}(\vec{\lambda})}{\partial \lambda_{j}} \\
& =\sum_{i=1}^{k}\left\{\frac{-X_{i}^{2}+n_{i}^{2} p_{i}^{2}(\vec{\lambda})}{n_{i} p_{i}^{2}(\vec{\lambda})}\right\} \cdot \frac{\partial p_{i} i(\vec{\lambda})}{\partial \lambda_{j}} \\
& =\sum_{i=1}^{k}\left(1-\left(\frac{f_{i}}{p_{i}(\vec{\lambda})}\right)^{2}\right) \cdot \frac{n_{i} \cdot \partial p_{i}(\vec{\lambda})}{\partial \lambda_{j}}
\end{aligned}
$$

So the minimum chi-square estimation $\tilde{\lambda}$ of $\vec{\lambda}$ is the solution to:

$$
\sum_{i=1}^{k}\left\{1-\left[\frac{f_{i}}{p_{i}(\vec{\lambda})}\right]^{2}\right\} \cdot \frac{n_{i} \cdot \partial p_{i}(\vec{\lambda})}{\partial \lambda_{j}}=0, \quad j=1,2, \cdots, s
$$

\section{INVERSE DATA EST IMATION METHOD BASED ON ISOT ONIC REGRESSION AND MINIMUM CHI-SQUARED EST IMATION}

a. We use PAVA algorithm to regulate the inverse in our data: select $\omega=\left(\omega_{1}, \cdots, \omega_{k}\right)^{\prime}, \omega_{i}>0$, then get the isotonic regression of $\left\{f_{i}=\frac{X_{i}}{n_{i}}\right\}$, which is $f_{i}^{*}$, then mark:

$$
X_{i}^{*}=f_{i}^{*} \cdot n_{i}
$$

b. Assuming that the overall theoretical failure rate $p_{i}(\vec{\lambda})$, which is at the time $t_{i}$, obeys specific distribution function $F(t, \vec{\lambda})$, in other words:

$$
p_{i}(\vec{\lambda})=F\left(t_{i}, \vec{\lambda}\right)
$$

c. Calculate the chi-square statistic:

$$
\chi^{2}(\vec{\lambda})=\sum_{i=1}^{k} \frac{\left(X_{i}^{*}-n_{i} p_{i}(\vec{\lambda})\right)^{2}}{n_{i} p_{i}(\vec{\lambda})}=\sum_{i=1}^{k} \frac{n_{i}\left(f_{i}^{*}-p_{i}(\vec{\lambda})\right)^{2}}{p_{i}(\vec{\lambda})}
$$

d. Solve the equation:

$$
\sum_{i=1}^{k}\left\{1-\left[\frac{f_{i}^{*}}{p_{i}(\vec{\lambda})}\right]^{2}\right\} \cdot \frac{n_{i} \cdot \partial p_{i}(\vec{\lambda})}{\partial \lambda_{j}}=0, \quad j=1,2, \cdots, s
$$


and we can get $\tilde{\lambda}$, which is the minimum chi-square estimation of $\vec{\lambda}$.

e. Do Pearson chi-square goodness of fit test. If the result meet with the requirement, end the calculation; if not, go back to step b.

\section{V. $\left(\tilde{\lambda}_{\mathrm{L}}, \tilde{\lambda}_{\mathrm{U}}\right)$, THE CONFIDENCE INTERVAL OF $\tilde{\lambda}$}

In practical engineering applications, we should be more concerned about the range of a reliability parameter in a certain probability, which is $\left(\tilde{\lambda}_{j \mathrm{~L}}, \tilde{\lambda}_{j \mathrm{U}}\right)$, the confidence interval of $\tilde{\lambda}_{j}$. Under the given confidence level $\alpha$, make $P\left(\tilde{\lambda}_{j \mathrm{~L}} \leq \tilde{\lambda}_{j} \leq \tilde{\lambda}_{j \mathrm{U}}\right)=1-\alpha$. After regulating the inverse data, we calculate:

$$
\frac{\partial \chi^{2}(\vec{\lambda})}{\partial \lambda_{j}}=\sum_{i=1}^{k} n_{i} \frac{\partial p_{i}(\vec{\lambda})}{\partial \lambda_{j}}\left[1-\left(\frac{f_{i}^{*}}{p_{i}(\vec{\lambda})}\right)^{2}\right]
$$

And function $\chi^{2}(\vec{\lambda})$ can get its min when $\vec{\lambda}=\tilde{\lambda}$. So when $j=1,2, \cdots$, we can always get:

$$
\begin{gathered}
\chi^{2}\left(\tilde{\lambda}_{j}\right) \leq \chi^{2}\left(\tilde{\lambda}_{j \mathrm{~L}}\right) \\
\chi^{2}\left(\tilde{\lambda}_{j}\right) \leq \chi^{2}\left(\tilde{\lambda}_{j \mathrm{U}}\right)
\end{gathered}
$$

Assuming $\chi^{2}\left(\tilde{\lambda}_{j \mathrm{~L}}\right)$ equals to $\chi^{2}\left(\tilde{\lambda}_{j \mathrm{U}}\right)$, the necessary and sufficient condition of $P\left(\tilde{\lambda}_{j \mathrm{~L}} \leq \tilde{\lambda}_{j} \leq \tilde{\lambda}_{j \mathrm{U}}\right)=1-\alpha$ is:

$$
P\left(\chi^{2}\left(\tilde{\lambda}_{j}\right) \leq \chi^{2}\left(\tilde{\lambda}_{j \mathrm{~L}}\right)=\chi^{2}\left(\tilde{\lambda}_{j \mathrm{U}}\right)\right)=1-\alpha
$$

From $\chi^{2}(\tilde{\lambda})=\sum_{i=1}^{k} \frac{\left(X_{i}^{*}-n_{i} p_{i}(\tilde{\lambda})\right)^{2}}{n_{i} p_{i}(\tilde{\lambda})} \sim \chi_{k-s-1}^{2}$, we know that:

$$
\chi^{2}\left(\tilde{\lambda}_{j \mathrm{~L}}\right)=\chi^{2}\left(\tilde{\lambda}_{j \mathrm{U}}\right)
$$

is the $(1-\alpha)$ quantile of $\chi_{k-s-1}^{2}$, which is:

$$
\chi^{2}\left(\tilde{\lambda}_{j \mathrm{U}}\right)=\chi^{2}\left(\tilde{\lambda}_{j \mathrm{~L}}\right)=\chi_{k-s-1}^{2}(1-\alpha)
$$

Then we can get $\left(\tilde{\lambda}_{j \mathrm{~L}}, \tilde{\lambda}_{j \mathrm{U}}\right)$, which is the confidence intervals of $\tilde{\lambda}_{j}$, by solving the equation.

\section{SIMULATION RESULT S AND ANALYSIS}

In order to investigate the effect of the parameter estimation method in this paper for estimating data, we conducted a simulation contract. Assuming the product storage life to obey two-parameter Weibull distribution:

$$
F(t)=1-\exp \left(-\left(\frac{t}{\eta}\right)^{m}\right)
$$

Compare the maximum likelihood estimation and the estimation method proposed in this paper which is based on is otonic regression and minimum chi-square theory. Conduct the destructive regular storage life test by the method below:

According to practical engineering experience, we mark $k$ as the group number of each test and choose $k=4, k=6, k=8$ respectively, the values of $\left\{\left(t_{i}, n_{i}\right)\right\}$ can be referred in Table 1 below:

TABLE I. THE VALUE OF $\left\{\left(\boldsymbol{t}_{i}, \boldsymbol{n}_{i}\right)\right\}$.

\begin{tabular}{|c|c|c|}
\hline \multicolumn{2}{|c|}{$k$} & $\left(t_{i}, n_{i}\right)$ \\
\hline \multicolumn{2}{|c|}{$k=4$} & $(8,12),(11,15),(13,10),(15,8)$ \\
\hline \multirow{3}{*}{$k=6$} & 1 & $(5,6),(8,7),(11,8),(13,6),(15,5),(18,8)$ \\
\cline { 2 - 4 } & 2 & $(5,10),(8,12),(11,15),(13,10),(15,8),(18,15)$ \\
\cline { 2 - 4 } & $(3)$ & $(5,30),(8,35),(11,40),(13,30),(15,25),(18,40)$ \\
\hline \multicolumn{2}{|c|}{$k=8$} & $(3,8),(5,10),(8,12),(11,15),(13,10),(15,8),(18,15),(20,12)$ \\
\hline
\end{tabular}

When $\eta=10, m=3$, simulating 100 times, according to the coefficient of variation of the estimated parameters, we assess the superiority of an estimation method, here is the coefficient of variation:

$$
C \cdot V(\zeta)=\frac{\sigma_{\zeta}}{\mu_{\zeta}}=\frac{\sqrt{D(\zeta)}}{E(\zeta)}=\frac{\sqrt{\frac{1}{l-1} \sum_{i=1}^{l}\left(\tilde{\zeta}_{i}-\zeta\right)^{2}}}{\frac{1}{l} \sum_{i=1}^{l} \tilde{\zeta}_{i}}
$$

\begin{tabular}{|c|c|c|c|c|c|}
\hline \multirow{2}{*}{\multicolumn{3}{|c|}{$\begin{array}{l}\text { Simulation } \\
\text { Results }\end{array}$}} & \multirow{3}{*}{$\begin{array}{c}\text { MLE } \\
0.341467\end{array}$} & \multicolumn{2}{|c|}{ Proposed Method } \\
\hline & & & & \multirow{2}{*}{$\begin{array}{c}\omega_{i}=n_{i} \\
0.254468\end{array}$} & \multirow{2}{*}{$\begin{array}{c}\omega_{i}=X_{i}\left(1-\frac{X_{i}}{n_{i}}\right) \\
0.250832\end{array}$} \\
\hline \multirow{5}{*}{$C \cdot V(\eta)$} & \multicolumn{2}{|c|}{$k=4$} & & & \\
\hline & \multirow{3}{*}{$k=6$} & (1) & 0.325576 & 0.198375 & 0.192727 \\
\hline & & (2) & 0.281259 & 0.224421 & 0.200703 \\
\hline & & (3) & 0.236022 & 0.197084 & 0.184062 \\
\hline & \multicolumn{2}{|c|}{$k=8$} & 0.286446 & 0.203605 & 0.201239 \\
\hline
\end{tabular}

in which, $\zeta$ can be referred as a parameter, which represents $\eta$ or $m$ here.

To make our conclusions more representative, we compare the coefficient of variation while $k$ and $\left(t_{i}, n_{i}\right)$ are changing. We can see the simulation results in Table 2 and 3.

TABLE II. COEFFICIENTOF VARIATION FOR DIMENSION PARAMETER $\eta$

TABLE III. COEFFICIENT OF VARIATION FOR SHAPE PARAMETER $m$

\begin{tabular}{|c|c|c|c|c|c|}
\hline \multicolumn{2}{|c|}{$\begin{array}{c}\text { Simulation } \\
\text { Results }\end{array}$} & \multirow{2}{*}{ MLE } & \multicolumn{2}{c|}{ Proposed Method } \\
\cline { 4 - 6 } & & $\omega_{i}=n_{i}$ & $\omega_{i}=X_{i}\left(1-\frac{X_{i}}{n_{i}}\right)$ \\
\hline \multirow{4}{*}{$C \cdot V(m)$} & $k=4$ & 0.150583 & 0.060764 & 0.060607 \\
\cline { 3 - 6 } & \multirow{3}{*}{$k=6$} & 1 & 0.137321 & 0.073290 & 0.068794 \\
\cline { 3 - 6 } & & $(2)$ & 0.134984 & 0.065273 & 0.065867 \\
\cline { 3 - 6 } & & $(3)$ & 0.129926 & 0.044917 & 0.040352 \\
\cline { 2 - 6 } & $k=8$ & 0.143542 & 0.053715 & 0.053082 \\
\hline
\end{tabular}

We can see that the traditional method of maximum likelihood estimation has a strict requirement for the size of the sample, to obtain a smaller coefficient of variation; we can only 
increase the sample size, which is difficult to carry out in practical engineering applications. The method proposed in this paper has a smaller coefficient of variation comparing to MLE while doing parameter estimation and the coefficient of variation changes little when the sample size changes. This is mainly because isotonic regression reduces the adverse effect of "inverse" data for parameter estimation, while minimum chisquare estimation can be more stable when dealing with the small sample data compared to maximu m likelihood estimation theory. When conducting the storage life test, by the method proposed in this paper, we can solve the problem that the sample size is too small by increasing the value of $k$ properly.

When conducting isotonic regression, we can set different weights $\omega_{i}$. In this paper we select $\omega_{i}=n_{i}$ and $\omega_{i}=X_{i}\left(1-X_{i} / n_{i}\right)$ to contrast. We can see that the coefficient of variation obtained by the second weight is smaller, because the second weight considers both the randomness of number of failures and the selection of samples. When we choose $\omega_{i}=n_{i}$, we can deal with the data more conveniently.

\section{ACKNOWLEDGMENT S}

This work is supported by the National Natural Science Foundation of China (Grant No. 61104133).

\section{REFERENCES}

[1] T. Robertson, F. T. Wright, and R. L. Dykstra. Order Restricted Statistical Inference. New York: John Wiley \& Sons, 1988.

[2] J. Berkson. Minimum Chi-squared, not Maximum Likelihood! The Annals of Statistics, 1980, 8(2): 457-487.

[3] N. Balakrishnan, E. Beutner, M. Kateri. Order Restricted Inference for Exponential Step-Stress Models. IEEE Transactions on Reliability, 2009, 58(1): 132-142.

[4] Quang H. Vuong. Minimum chi-square estimation and tests for model selection. Journal of Econometrics, 1993, 56(1-2): 141-168.

[5] H. M. Fu. Method for Reliability Assessment with Very Small Sample. Journal of Mechanical Strength, 2005, 27 (3): 335-338.

[6] N. Z. Shi. Isotonic Regression and Maximum Likelihood Estimation. Applied Probability and Statistics, 1993, 9(2): 203-214.

[7] N. Z. Shi. Maximum likelihood estimation of means and variances from normal populations under simultaneous order restrictions. Journal of Multivariate Analysis, 1994, 50(2): 282-293.

[8] R. T. Zhang, H. X. Liu. A Method for Estimating "Inverse" Data in Statistical Analysis of Reliability, 1991, 4(4): 22-28.

[9] B. J. Song, Y. X. Qin. A Reliability Assessment Method for small sample of products based on Bayes. Missiles and Guidance, 1999, 20(2): 52-57.

[10] T. A. Mazzuchi, R. Soyer. A Bayes method for assessing productreliability during development testing. IEEE Trans. Reliability, 42(18): 503-510. 